\title{
Fermiology of Cuprates from First Principles: From Small Pockets to the Luttinger Fermi surface
}

\author{
L. Hozoi, M. S. Laad and P. Fulde \\ Max-Planck-Institut für Physik komplexer Systeme, Nöthnitzer Str. 38, 01187 Dresden, Germany
}

(Dated: August 18, 2021)

Fermiology, the shape and size of the Fermi surface, underpins the low-temperature physical properties of a metal. Recent investigations of the Fermi surface of high- $T_{c}$ superconductors, however, show a most unusual behavior: upon addition of carriers, "Fermi" pockets appear around nodal (hole doping) and antinodal (electron doping) regions of the Brillouin zone in the "pseudogap" state. With progressive doping, $\delta$, these evolve into well-defined Fermi surfaces around optimal doping $\left(\delta_{\text {opt }}\right)$, with no pseudogap. Correspondingly, various physical responses, including $d$-wave superconductivity, evolve from highly anomalous, up to $\delta_{\text {opt }}$, to more conventional beyond. Describing this evolution holds the key to understanding high-temperature superconductivity. Here, we present ab initio quantum chemical results for cuprates, providing a quantitative description of the evolution of the Fermi surface with $\delta$. Our results constitute an ab initio justification for several, hitherto proposed semiphenomenological theories, offering an unified basis for understanding of various, unusual physical responses of doped cuprates.

Understanding high-temperature superconductivity in quasi two-dimensional (2D), doped copper oxides remains one of the most challenging problems in condensed matter physics. In spite of varying structural and chemical details, the phase diagram of the high- $T_{c}$ superconductors (HTS's) is seemingly remarkably universal: the undoped compounds with nominally one hole per $\mathrm{Cu}$ site are Mott insulators (MI's) due to strong electron-electron interactions [1]. Upon addition of charge carriers (doping), the cuprates turn into $d$-wave superconductors ( $d$ $\mathrm{SC})$ at low temperatures, $T<T_{c}[1]$.

The "normal" state for $T>T_{c}$ is actually very abnormal, and radically undermines the conventional Landau theory of Fermi liquids (FL's). In the so-called underdoped (UD) regime, $\delta \ll 1$, a $d$-wave pseudogap $(d$-PG) characterises the normal state [1]. Whether this $d-\mathrm{PG}$ state is the precursor of $d$-SC at lower $T$ or its competitor is hotly debated [2, 3]. Around optimal doping, a "strange metal" phase, with most unusual singular responses [4] is clearly revealed: this is the celebrated nonFL metallic state that has been investigated for twenty years [1]. In the overdoped (OD) regime $\left(\delta>\delta_{\text {opt }}\right)$, low- $T$ FL behavior seems to be smoothly recovered.

Very recently, notable improvements in sample quality as well as measuring technology have finally allowed accurate mapping of the actual dispersion of the quasiparti- cles (QP's) and the Fermi surface (FS) of HTS's. Specifically, angle-resolved photoemission (ARPES) 5, 6, 7] and quantum oscillation $(\mathrm{SdH})$ techniques [8, 9] reveal crucial, hitherto unmapped features of the evolution of the (renormalised) QP dispersion as a function of doping. Thus, these works open up the possibility, for the first time, of unearthing the link between the electronic structure and physical responses of cuprates in microscopic detail as a function of $\delta$. Both ARPES and SdH measurements reveal a full FS consistent with conventional band-structure calculations for $\delta>\delta_{\text {opt }}[5,[10]$. However, in the UD regime, the small "Fermi" pockets inferred by SdH experiments are in deep conflict with Luttinger's theorem. For hole doped samples with $\delta=0.1$, the $\mathrm{SdH}$ results yield a carrier concentration $x_{\mathrm{SdH}}=0.15$ [8]. Under the same conditions, the low- $T$ Hall constant is electron-like [11]. How can this come about? Existing theoretical calculations cannot resolve this issue satisfactorily. And yet, this finding points toward a glaring discrepancy in our understanding of the electronic structure of cuprates. In light of these findings, a consistent theoretical scenario aiming to describe the unique physics of HTS's must now base itself upon the appropriate, collective excitations stemming from the observed, detailed shape and size of the FS.

Here, we study the dispersion of the lowest hole and electron-addition states, as well as the evolution of the renormalised FS with doping. Using an ab initio wavefunction-based formalism, we describe these with quantitative accuracy vis-a-vis recent ARPES and SdH measurements. Implications of our findings for other experiments, as well as their connection to earlier modelbased and semiphenomenological theories, are discussed in detail. Our findings lend credence to the view [12], that the unique properties of cuprates are those of a $2 \mathrm{D}$, doped MI.

\section{THEORETICAL FRAMEWORK}

The correlation-induced renormalisation effects on the valence and conduction energy bands are remarkably strong in cuprates. Early attempts to describe these effects were based on the $t$ - $J$ model [1] and indicated the crucial role played by the strong antiferromagnetic (AF) couplings in reducing the effective bandwidths. In the three-band context, it was suggested that a doped oxygen hole would induce short-range ferromagnetic (FM) 
correlations between adjacent $\mathrm{Cu}$ sites [13]. If the extra hole delocalizes over all four equivalent ligands of a given $\mathrm{CuO}_{4}$ plaquette 14], these $\mathrm{FM}$ correlations would involve $\mathrm{Cu}$ sites on five plaquettes [15], as shown in Fig. 1. Since the mobility of this entity is expected to be small, it is often referred to as a FM "spin polaron".

An accurate investigation of the structure of such composite objects calls for methods that allow an unbiased treatment of the various (competing) interactions in the $\mathrm{CuO}_{2}$ plane. A fundamental point underlying the physics of cuprates is the interplay between electron localization effects as a result of strong repulsive interactions and band-like behaviour as a result of translational symmetry and inter-site orbital overlap. Standard band theories based upon the density-functional model (and the local density approximation, LDA) mainly emphasize the latter aspect. Though LDA provides rather good results for weakly correlated solids, its limited ability to describe correlated $d$ (and $f$ ) electrons is well-documented. With the advent of dynamical mean-field theory (DMFT), this basic conflict has been partially resolved [16]. In particular, much progress in describing the FS's of real materials, along with their one-particle spectral functions, has been possible. However, this is still some distance from being a totally $a b$ initio approach, since the actual correlations are approximated by local (Hubbard) parameters. Use of constrained LDA to estimate these parameters entails an uncertainty of the order of $20 \%$, while their self-consistent estimation within LDA+DMFT is fraught with insurmountable problems [17].

An alternative approach bases itself on state-of-the-art quantum chemical (QC) methods [18]. In molecular systems, wavefunction-based quantum chemistry provides a rigorous theoretical framework for addressing the electron correlation problem [19]. A real-space, QC-based treatment is then a natural starting point in dealing with

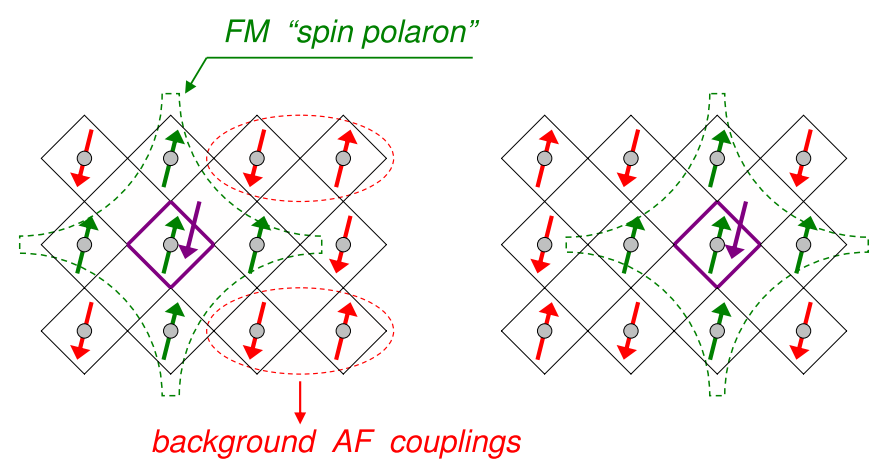

FIG. 1: Ferromagnetic correlations (green) among $\mathrm{Cu}$ sites around a doped oxygen hole (violet) as inferred from CASSCF calculations. Farther spin couplings are antiferromagnetic (red). The motion of the oxygen hole is coherent when the FM spin polarization "cloud" at adjacent $\mathrm{Cu}$ sites moves solidarily with the hole. The $\mathrm{O}$ ligands are at the vertices of the square plaquettes, the $\mathrm{Cu}$ ions are shown as grey dots.
Mott physics in $d$-metal solid state compounds. As shown below, the $\mathbf{k}$-dependent energy bands can be recovered at a later stage after rigorously accounting for the ubiquitous strong short-range correlation effects.

The strategy is to use a sufficiently large cluster, $\mathcal{C}$, cut out from the infinite solid and properly embedded in some effective lattice potential, capable of describing these crucial short-range correlations accurately. The presence of partially filled $d$ electron shells requires a multiconfiguration representation of the many-electron wavefunction. The complete-active-space (CAS) selfconsistent-field (SCF), CASSCF, method 20] provides precisely such a framework (see Methods for details). It can, for example, describe spin correlation effects such as the Anderson superexchange in MI's 21 and the doubleexchange in mixed-valence systems [22]. For undoped cuprates, with formally one $3 d_{x^{2}-y^{2}}$ electron per $\mathrm{Cu}$ site, the CAS wavefunction is similar to the variational wavefunction used in numerical studies of the $2 \mathrm{D}$, one-band Hubbard model 23]. However, all one- and two-particle integrals are computed here in a totally $a b$ initio way.

The dispersion of $d$-like states on a square lattice is given by the following relation:

$$
\begin{aligned}
\epsilon(\mathbf{k})= & -2 t\left(\cos k_{x} a+\cos k_{y} a\right)+4 t^{\prime} \cos k_{x} a \cos k_{y} a \\
& -2 t^{\prime \prime}\left(\cos 2 k_{x} a+\cos 2 k_{y} a\right)
\end{aligned}
$$

where $t, t^{\prime}, t^{\prime \prime}$ are the hopping integrals between nearestneighbor $(\mathrm{NN})$, second-NN and third-NN sites and the effective site is one $\mathrm{CuO}_{4}$ plaquette [14]. LDA calculations yield $t$ values of $0.4-0.5 \mathrm{eV}$ and a ratio between the $\mathrm{NN}$ and second-NN hoppings $t^{\prime} / t \approx 0.15$ for $\mathrm{La}_{2} \mathrm{CuO}_{4}$ and 0.33 for $\mathrm{Tl}_{2} \mathrm{Ba}_{2} \mathrm{CuO}_{6}$ [24]. In contrast, the CASSCF calculations predict a renormalised $\mathrm{NN}$ hopping $t=0.135$ $\mathrm{eV}$ in $\mathrm{La}_{2} \mathrm{CuO}_{4}$ 15]. Here, we describe how the detailed QP dispersion can be obtained with quantitative accuracy, for both hole and electron-addition states. The effective hoppings are computed by using the overlap, $S_{i j}$, and Hamiltonian, $H_{i j}$, matrix elements between $(N \mp 1)$-particle wavefunctions having the additional particle (hole or electron) located on different plaquettes $(i, j, \ldots)$ of a given cluster. Each of these $(N \mp 1)$ wavefunctions, $\left|\Psi_{i}^{N \mp 1}\right\rangle$, is obtained by separate CASSCF optimizations. A similar scheme was previously applied to simpler, noncontroversial systems such as diamond, silicon and $\mathrm{MgO}$ 25, 26]. It accounts for both charge 25, 26] and spin polarization and relaxation effects in the nearby surroundings, see Fig. 1. For degenerate (i.e., $H_{i i}=H_{j j}$ ) $(N \mp 1)$ states, $t=\left(\epsilon_{j}-\epsilon_{i}\right) / 2=\left(H_{i j}-S_{i j} H_{i i}\right) /\left(1-S_{i j}^{2}\right)$, where $\epsilon_{i}$ and $\epsilon_{j}$ are the eigenvalues of the $2 \times 2$ secular problem. For non-degenerate $\left(H_{i i} \neq H_{j j}\right)$ states, $t=1 / 2\left[\left(\epsilon_{j}-\epsilon_{i}\right)^{2}-\left(H_{j j}-H_{i i}\right)^{2}\right]^{1 / 2}$. The $S_{i j}$ and $H_{i j}$ terms are computed using the State-Interaction (SI) method 27]. Correlation effects beyond CASSCF on the onsite matrix elements $H_{i i}$ are calculated by multiconfigurational second-order perturbation theory, CASPT2 
[28]. The short-range magnetic correlations are included in our clusters by adding extra $\mathrm{CuO}_{4}$ units around those plaquettes directly involved in the hopping process, see Fig. 2.

\section{QUASIPARTICLE BANDS AND ARPES}

We investigated both, the $p$-type $\mathrm{HTS} \mathrm{La}_{2} \mathrm{CuO}_{4}$ and the $n$-type $\mathrm{HTS} \mathrm{SrCuO}_{2}$. Renormalised hopping matrix elements (ME's) for Zhang-Rice (ZR) [14] type states in $\mathrm{La}_{2} \mathrm{CuO}_{4}$ and electron-addition states in $\mathrm{SrCuO}_{2}$, involving neighbors up to the third order, are listed in Table I. For comparison, "unrenormalised" (or bare) hoppings were also computed, by imposing a FM arrangement of spins at the nearby $\mathrm{Cu}$ sites, i. e., a FM lattice. The bare hoppings are substantially smaller for the $(N+1) \mathrm{Cu} d^{10}$ states because the $\mathrm{Cu} 3 d$ functions are more compact as compared to the $\mathrm{O} 2 p$ orbitals.

In effective one-band models [1, 14], the ZR $p$ - $d$ state is regarded as a vacant, or unoccupied, $d$-like site. Consequently, there is no renormalisation of the second-NN and third-NN hoppings $t^{\prime}$ and $t^{\prime \prime}$ because these connect sites of the same magnetic sublattice. In contrast, the interplay between short-range FM correlations and longerrange AF couplings (see the sketch in Fig. 1) produces large renormalisation effects for all hopping ME's in our approach. For $t^{\prime}$ and $t^{\prime \prime}$, in particular, the hopping of the $2 p$ hole implies coupled, $\mathrm{Cu}$ and $\mathrm{O}$ spin "flips" on the two plaquettes directly involved in the hopping process. Nevertheless, spin correlations decay rapidly with distance and so the renormalisation effects are less drastic for the third-NN ME, $t^{\prime \prime}$. We thus find $t^{\prime \prime} \simeq t / 2$ and $t^{\prime} \ll t$, a rather remarkable result.

For the description of the electron-addition $d^{10}$ states, an effective one-band model is seen to be justified. As

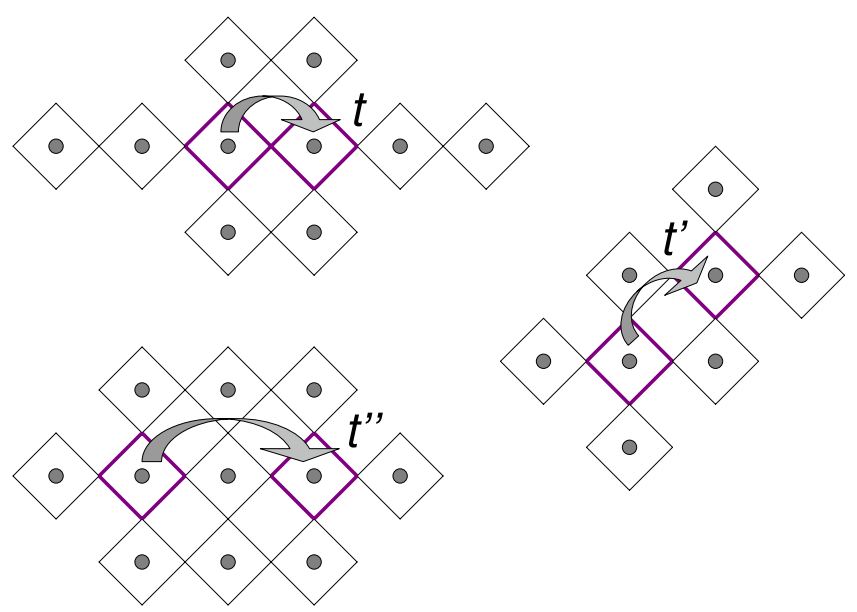

FIG. 2: Sketch of the finite clusters employed for the calculation of the effective valence-band and conduction-band hoppings. shown in Table I, in this case only $t$ is substantially affected by nonlocal spin correlations. Hence, for the $(N+1)$ states, the renormalized hoppings satisfy $t^{\prime} \simeq t$, an equally remarkable result. The particle-hole asymmetry, see Fig. 3, is now readily understood from the very different $t^{\prime} / t$ and $t^{\prime \prime} / t$ values for the $(N-1)$ (ZR-like) and $(N+1)\left(\mathrm{Cu} d^{10}\right)$ bands.

Doping of the $\mathrm{CuO}_{2}$ planes is achieved by chemical substitution in the "reservoir" layers. The dopant carriers must quantum mechanically tunnel from the reservoir to the planes: this necessarily involves the apical $\mathrm{O} 2 p_{z}, \mathrm{Cu}$ $3 d_{z^{2}}$ and $\mathrm{Cu} 4 s$ orbitals, causing additional renormalisation of the planar QP's from these apical charge-transfer interactions. Hence, we extended our calculations to include configurations where an electron is removed/added from/to the $\mathrm{Cu} 3 d_{z^{2}}$ or $\mathrm{Cu} 4 s$ orbital. Only the $\mathrm{Cu} 3 d_{z^{2}}$ $(N-1)$ state gave rise to considerable renormalisation of the planar QP dispersion. While the onsite mixing between the ZR and $d_{z^{2}}(N-1)$ configurations and NN hopping between degenerate $d_{z^{2}}$ hole states are negligible, the inter-site off-diagonal hopping is large. With sets of orbitals individually optimized for each $(N-1)$ state, this off-diagonal ME is $t_{m}=0.20 \mathrm{eV}$, larger than the value reported in ref. [30], where the lowest $d_{z^{2}}$ hole state was expressed in terms of orbitals optimized over an average of several excited states involving different couplings among the nearby $\mathrm{Cu}$ spins. In $\mathbf{k}$-space, the hybridisation ME between the ZR and $d_{z^{2}}$ bands reads $\gamma_{m}(\mathbf{k})=t_{m}\left(\cos k_{x} a-\cos k_{y} a\right)$. Further, onsite, the ZR and $d_{z^{2}}(N-1)$ states are separated by an energy $\Delta \epsilon$. It turns out that the correlation-induced corrections to the CASSCF energy separation are substantial, changing this quantity from $0.60 \mathrm{eV}$ [30] to $\Delta \epsilon=1.70$ at the CASPT2 level. Such corrections are usually small, for both the onsite relative energies [21] and hoppings [15, 26]. Nevertheless, corrections as large as $0.9 \mathrm{eV}$ have been found before for the relative energy of the ${ }^{1} A_{1 g}$ state of the $d^{8}$ manifold in $\mathrm{NiO}$ [21], for example.

It is now straightforward to diagonalise the $\mathbf{k}$ dependent $2 \times 2$ matrix,

$$
\left(\begin{array}{cc}
\epsilon_{\mathrm{ZR}}(\mathbf{k}) & \gamma_{m}(\mathbf{k}) \\
\gamma_{m}(\mathbf{k}) & \epsilon_{z^{2}}(\mathbf{k})+\Delta \epsilon
\end{array}\right)
$$

to yield the renormalised bands. This constitutes a nontrivial extension of the three-band Hubbard model, where the additional renormalisation from the apical link is not considered. The resulting dispersion of the ZR-like band is plotted in Fig. 3 and shows excellent agreement with the dispersion of the lowest ARPES band reported for $\mathrm{La}_{2} \mathrm{CuO}_{4}$ by Ino et al. [31]. In particular, the flat dispersion around $(0, \pi)$, the maximum near $(\pi / 2, \pi / 2)$ and a renormalized bandwidth of nearly $1 \mathrm{eV}$ are all faithfully reproduced in the theoretical results. We have not attempted to describe the "waterfall"-like structures observed recently in ARPES. First, their interpretation 32. and causal link to $d$-SC are controversial. Theoretically, 
TABLE I: Hopping ME's for ZR-like states in $\mathrm{La}_{2} \mathrm{CuO}_{4}$ and electron-addition $\mathrm{Cu} d^{10}$ states in $\mathrm{SrCuO}_{2}$. The bare hoppings were computed by imposing high-spin couplings among the nearby $\mathrm{Cu}$ sites. For the $d^{10}$ states, each of the hoppings changes by less than $15 \%$ from $\mathrm{La}_{2} \mathrm{CuO}_{4}$ to $\mathrm{SrCuO}_{2}$ (not shown in the table). On the other hand, no ZR-like solution was obtained for the $(N-1)$ states in $\mathrm{SrCuO}_{2}$, which qualitatively confirms the experimental findings: for in-plane lattice constants $a \geq 3.87 \AA\left(a=3.925 \AA\right.$ in $\left.\mathrm{SrCuO}_{2}\right)$, the $\mathrm{CuO}_{2}$ planes do not readily accept holes in the $\mathrm{O}$ bands [29].

\begin{tabular}{lcc}
\hline \hline Hopping ME's & Bare & Renormalised \\
\hline "ZR" state & & \\
$t$ & & \\
$t^{\prime}$ & 0.540 & 0.135 \\
$t^{\prime \prime}$ & 0.305 & 0.010 \\
& 0.115 & 0.075 \\
$d^{10}$ state & & \\
$t$ & & \\
$t^{\prime}$ & 0.290 & 0.115 \\
$t^{\prime \prime}$ & 0.130 & 0.130 \\
\hline \hline
\end{tabular}

the study of such structures requires an analysis of the incoherent part of the spectral function [16, 33]. This challenging exercise is beyond the scope of our present work. For the $\mathrm{Cu} d^{10}$ states, a lack of detailed data for ARPES lineshapes in $n$-type cuprates precludes a direct comparison between theory and experiment.

Knowledge of the QP dispersion enables us to study the evolution of the renormalized FS as a function of doping. Assuming a rigid band shift with doping, an assumption supported by independent experiments [34, 35], we plot the evolution of the FS for both hole and electron doped cuprates in Fig. 4. We simulate doping effects by a progressive downward shift (hole doping) and upward shift (electron doping) of the Fermi energy, $E_{F}$. Once again, our results show a remarkable agreement with the experiment: small hole pockets centered around the nodal $(\mathrm{N})$ region $\left[\mathbf{k}_{n}=(\pi / 2 a, \pi / 2 a)\right]$ comprise the "FS" in the deeply UD regime [8, 9]. Additionally, for slightly higher $\delta$, smaller, electron-like pockets centred around the corners of the Brillouin zone are also clearly resolved, see Fig. 4(a). With further doping, these progressively evolve into a large hole-like FS [5], implying a FS reconstruction close to $\delta_{\text {opt }}$. At a critical value $\delta=\delta_{c}$, the FS changes from hole-like $\left(\delta<\delta_{c}\right)$ to electron-like $\left(\delta>\delta_{c}\right)$, in complete accord with results from ARPES 5]. Excellent agreement of the FS vis-a-vis experiment [36] is also obtained for the $n$-type cuprates: small pockets centered around $\mathbf{k}_{a n}$ evolve into a hole-like FS with progressive electron doping. To our knowledge, ours are the first $a b$ initio results capturing such effects: hitherto, these have been (partially) described within effective, one- [16, 33, 37] or three-band [38, 39] models with parametrized couplings.

\section{BROADER IMPLICATIONS}

Our results constitute an $a b$ initio derivation of the "hot-spot-cold-spot" phenomenology [40], also seen in cluster-DMFT work on the 2D Hubbard model [16]. There, the QP scattering rate is strongly k-dependent as the FS is traversed. This is mainfest in our computed FS: the pronounced dispersion of the N-QP's implies weaker QP scattering around $\mathbf{k}_{n}$, in marked contrast to the antinodal (AN) region, where strong band flattening is indicative of strong QP scattering and very short QP lifetimes. Further, the renormalized $t, t^{\prime}, t^{\prime \prime}$ imply intrinsically frustrated hopping: interestingly, the importance of frustrated kinetic energy to the high- $T_{c}$ problem has been discussed at length in the resonating valence bond (RVB) model of Anderson [12]. We show that both these seemingly disparate features arise from the same underlying microscopic mechanism: strongly anisotropic renormalisation of carrier motion by strong, short-range spin correlations.

Interestingly, in a Hubbard-type model, large $t^{\prime}$ (or $\left.t^{\prime \prime}\right)$ open the door to additional exotic phases, like $d$ wave nematic [41], $d$-density wave [42] and valence-bond 43] ordered phases. These have been invoked as possible competitors of $d$-SC in various semiphenomenological contexts. Our work establishes the intimate connection between these putative instabilities and short-range spin correlations characteristic of a (lightly doped) MI.

Our findings have remarkable implications for the interpretation of a host of experiments probing the un-
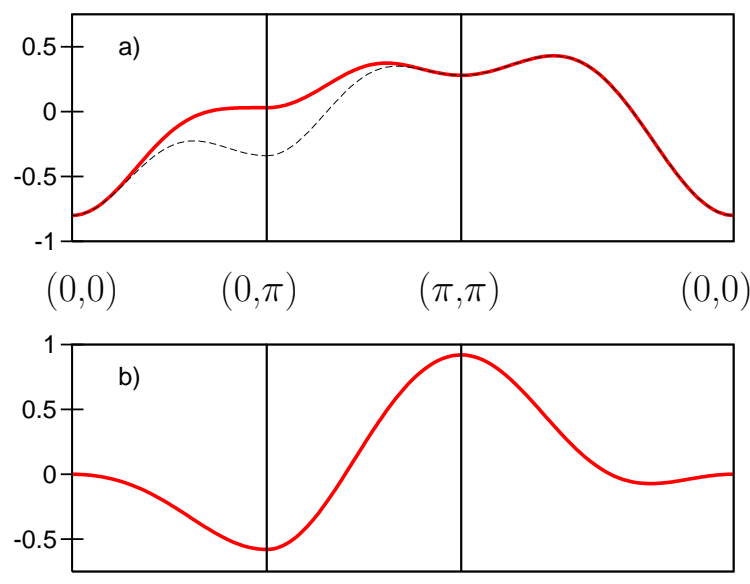

FIG. 3: (a): The ZR-like electron-removal band for $\mathrm{La}_{2} \mathrm{CuO}_{4}$ in the $2 \mathrm{D}$ Brillouin zone, without including the interaction with the $d_{3 z^{2}-r^{2}}$ hole state (dashed line) and after including this interaction (thick red line). For clarity, the $d_{3 z^{2}-r^{2}}$ band is not shown in the figure. The zero of energy is the value of the onsite Hamiltonian ME of the ZR state, $H_{i i}^{\mathrm{ZR}}$. (b): QP dispersion for the electron-addition $\mathrm{Cu} d^{10}$ state in $\mathrm{SrCuO}_{2}$. The reference energy is the value of the onsite Hamiltonian $\mathrm{ME}, H_{i i}^{d^{10}}$. Units of $\mathrm{eV}$ are used in both panels. 

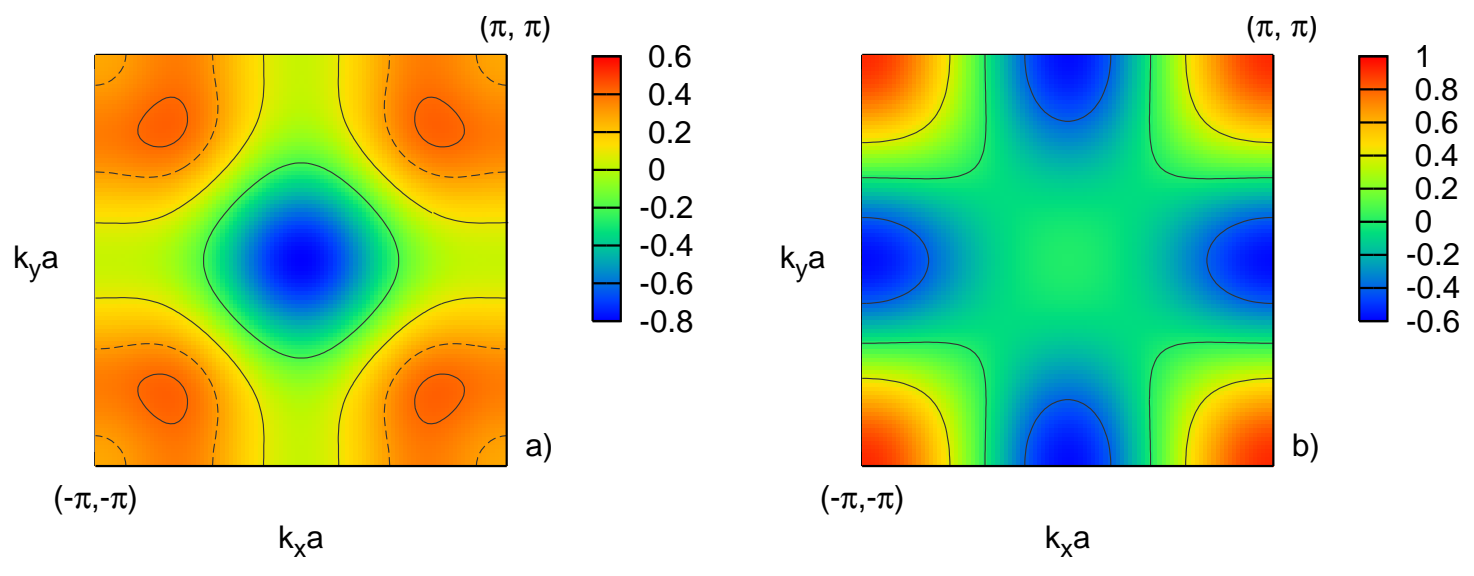

FIG. 4: (a): 2D colour map for the ZR-like QP dispersion in $\mathrm{La}_{2} \mathrm{CuO}_{4}$, including the effect of the $\mathrm{ZR}-d_{z^{2}}$ hole interaction. In a rigid-band picture, the constant-energy (CE) contours (black curves) illustrate the evolution of the FS with hole doping, from small (hole) pockets in the nodal region for deeply UD samples to a large, hole-like FS at intermediate dopings and an electron-like shape in the OD regime [5]. For a narrow doping interval in the UD regime, the "FS" is defined by eight contours (see the dashed curves), with both hole-like sheets and electron pockets centred at the corners of the Brillouin zone. The latter are related to the small dip in the dispersion at the $(\pi, \pi)$ point, see Fig. 3(a), and have been also inferred from Hall-effect measurements [1]]. (b): Colour map for the electron-addition $\mathrm{Cu} d^{10}$ states in $\mathrm{SrCuO}_{2}$. The CE contours reproduce the experimentally observed evolution of the FS with electron doping, from small electron pockets in the antinodal region for the lightly doped regime to a large, hole-like FS at high doping [5, 36]. The same energy scales as in Fig. 3 are used.

usual "normal"-state physical responses of the cuprates as a function of doping. First, the UD $p$-type cuprates are revealed to be $\mathrm{N}$-metals for small $\delta$, as inferred by ARPES [6, 7]. All the "nodal metal" phenomenology [1] can now be provided an ab initio justification in light of our results. Coherent nodal QP's also naturally explain the "good metal" thermal conductivity in the UD region [44]. Additionally, the $\mathrm{SdH}$ quantum oscillation frequencies $\Omega$ [8, 9] now correspond to coherent carrier orbits in the "pockets". The Onsager-Lifshitz formula, $\Omega=\Phi_{0} A /(2 \pi)^{2}$, with $\Phi_{0}=h c / e$ the flux quantum and $A=2 \pi^{2} x / 4 a^{2}$ the area of the pocket for $x$ carriers in $\mathbf{k}$-space, tells us that $\Omega(x) \sim x$. Our finding of additional electron-like pockets around $(\pi, \pi)$ in the hole doped case offers a route toward a resolution of one of the central controversies surrounding recent $\mathrm{SdH}$ experiments, where $x_{\mathrm{SdH}}=0.15$ for the $\delta=0.10$ cuprates [8]. While this is irreconcilable with theories having only hole pockets, our finding of additional electron-like sheets can reconcile the $\mathrm{SdH}$ results with the Luttinger sum rule for $\delta \approx 0.1[\underline{8}, 9]$. Moreover, the Hall constant $R_{H}(x)$ is now expected to track the evolution of the renormalised FS with doping. Depending upon the concentrations of hole and electron carriers $n_{h}$ and $n_{e}$, with $n_{h}>n_{e}$, and the mobilities $\mu_{h}$ and $\mu_{e}$ [11], $R_{H}$ may change sign from hole-like to electron-like with $T$, reconciling the $\operatorname{SdH}[8,9]$ and Halleffect [11] data.

Finally, what about superconductivity? Given the small number, $\delta$, of nodal QP's in the UD regime, $d$-SC will result from pairing of these quasicoherent fermionic entities. Without going into the nature of the pairing mechanism, the above implies that $T_{c}(\delta) \sim \delta$ for $\delta \ll 1$. So the superfluid density at $T=0$ will decrease linearly as $\delta$ is reduced, implying that upon underdoping $d$-SC will be progressively destroyed by order parameter phase fluctuations, which grow as the MI is approached. This implies non-BCS (large) values of $2 \Delta / k_{B} T_{c}$ and strong vortexliquid like effects above $T_{c}$ for UD cuprates. These have indeed been invoked in connection with the anomalous, "giant" Nernst effect in UD superconductors [2]. However, once a full FS develops, $d$-SC would be expected to revert back to a more conventional BCS-like variety [45], with identical scaling for the $\mathrm{N}$ and $\mathrm{AN}$ gaps.

\section{CONCLUSIONS}

To summarize, we have implemented a first-principles, wavefunction-based calculation of correlated hole and electron-addition quasiparticle states in layered cuprates. In addition to quantitatively describing the dispersion of the ZR-like band, our work reproduces the FS evolution as a function of doping, in remarkable agreement with a host of recent ARPES and quantum oscillation experiments. Our finding of large longer-range effective hoppings implies intrinsically frustrated carrier kinetic energy, in agreement with Anderson's RVB ideas [12]. The very different behavior of hole and electron doped cuprates is clearly manifested as originating from very different quantum chemical and spin correlation "backgrounds". Seen from this perspective, the FS "reconstruction" with doping [11], as well as the famed nodalantinodal dichotomy in the UD systems, are both un- 
derstood in terms of $\mathbf{k}$-space differentiation of QP states in the 2D, doped MI. Phenomenologically, the computed evolution of the FS with $\delta$ goes hand-in-hand with the observed evolution of $d$-SC from a strongly non-BCS, phase fluctuation dominated type, to a more conventional BCS type with progressive doping, benchmarking the crucial relevance of fermiology in cuprates.

\section{- Methods -}

In the CASSCF approach [19, 20], the wavefunction is written as a linear combination of configuration state functions (CSF's) $|m\rangle,|\Psi\rangle=\sum_{m} C_{m}|m\rangle$. These CSF's are spin(and symmetry-) adapted combinations of Slater determinants (SD's), i. e., eigenfunctions of the operators for the projected and total spins. In turn, the SD's are constructed from a set of real and orthonormal spin orbitals $\left\{\phi_{p}(\mathbf{r}, \sigma)\right\}$, where $\mathbf{r}$ and $\sigma$ are the spatial and spin coordinates, respectively. In this work, an initial guess for these orbitals is obtained from a Hartree-Fock calculation for an hypothetical $\mathrm{Cu} 3 d^{10}$, O $2 p^{6}$ closed-shell configuration of the $\mathrm{Cu}$ and $\mathrm{O}$ species.

In determining the CASSCF wavefunction, the orbitals are variationally optimized simultaneously with the coefficients of the CSF's. The orbitals employed for expressing the wavefunction are thus the optimal orbitals for the state at hand and do not introduce a bias toward a particular configuration. Three different sets of orbitals are used in CASSCF: (i) the inactive levels, doubly occupied in all configurations, (ii) the virtual orbitals, unoccupied in all configurations, and (iii) the active orbital set, where no occupancy restrictions are imposed. For undoped cuprates, with formal $\mathrm{Cu} 3 d^{9}$ and O $2 p^{6}$ valence states, the active space would include the partially occupied in-plane $\mathrm{Cu} 3 d_{x^{2}-y^{2}}$ orbitals. Such a CAS wavefunction is similar to the variational wavefunction used in numerical studies of the 2D, one-band Hubbard model [23]. The main difference is that all integrals, including inter-site Coulomb and exchange terms, are computed here in a totally $a b$ initio way. In particular, the lower, completely filled levels, e. g., the $\mathrm{O} 2 s$ and $2 p$ orbitals, do affect (i.e., screen) the actual interactions among the active electrons by readjusting themselves to fluctuations within the active orbital space.

If extra holes are created, the active space must be enlarged with orbitals from the inactive group. Each additional doped hole requires one orbital to be transferred from the inactive to the active space. For the lowest electron-removal state, for example, the orbital added to the active space turns into a ZR-type $p$ - $d$ composite [14] in the variational calculation, localized on a given $\mathrm{CuO}_{4}$ plaquette. With regard to the electron-addition conduction-band states, these turn out to have $\mathrm{Cu} 3 d^{10}$ character and, in a first approximation, an active orbital space including only the $3 d_{x^{2}-y^{2}}$ levels would suffice.

So-called dynamic correlation effects [19] for the onsite matrix elements $H_{i i}$ were computed using second-order perturbation theory (the CASPT2 method [28]). The $\mathrm{Cu} 3 d$ and O $2 s, 2 p$ electrons on five plaquettes (i.e., the ZR plaquette and the two apical ligands for that plaquette plus the four NN plaquettes) were correlated. All calculations were performed with the QC software MOLCAS [46]. For the ions of the plaquettes directly involved in the hopping process, allelectron basis sets (BS's) of triple-zeta quality were applied.
These were Gaussian-type atomic-natural-orbital BS's from the MOLCAS library [46], with the following contractions [19]: $\mathrm{Cu}(21 s 15 p 10 d) /[5 s 4 p 3 d]$ and $\mathrm{O}(14 s 9 p) /[4 s 3 p]$. The core electrons of the remaining ions of each cluster, see Fig. 2, were represented by effective core potentials (ECP's), i.e., $\mathrm{Cu}$ ECP's plus valence double-zeta BS's 47] and O ECP's with triple-zeta BS's [48]. To describe the finite charge distribution at the sites in the immediate neighborhood of the cluster, we model those ions by effective ion potentials [49]. Beyond these neighbors, we use large arrays of point charges (PC's) that reproduce the Madelung field within the cluster region. Apical ligands are explicitly included in our calculations only for the "active" plaquettes. Other apex oxygens are represented by formal PC's. That the charge populations of the $\mathrm{Cu} 3 d$ and active $\mathrm{O} 2 p$ orbitals are not sensitive to the size and shape of the clusters we use was shown in ref. [15]. We employed the crystal structure measured by Cava et al. [50] for $\mathrm{La}_{2} \mathrm{CuO}_{4}$ and by Smith et al. [29] for $\mathrm{SrCuO}_{2}$.

[1] Lee, P. A., Nagaosa, N. \& Wen, X.-G., Doping a Mott insulator: physics of high-temperature superconductivity. Rev. Mod. Phys. 78, 17 (2006).

[2] Wang, Y., Li., L \& Ong, N. P., Nernst effect in high- $T_{c}$ superconductors. Phys. Rev. B 73, 024510 (2006).

[3] Varma, C. M., Theory of the pseudogap state of the cuprates. Phys. Rev. B 73, 155113 (2006).

[4] van der Marel, D. et al., Quantum critical behaviour in a high- $T_{c}$ superconductor. Nature 425, 271-274 (2003).

[5] Damascelli, A., Hussain, Z. \& Shen, Z.-X., Angleresolved photoemission studies of the cuprate superconductors. Rev. Mod. Phys. 75, 473-541 (2003).

[6] Tanaka, K. et al., Distinct Fermi-momentum-dependent energy gaps in deeply underdoped Bi2212. Science 314, 1910-1913 (2006).

[7] Kanigel, A. et al., Evolution of the pseudogap from Fermi arcs to the nodal liquid. Nature Physics 2, 447451 (2006).

[8] Doiron-Leyraud, N. et al., Quantum oscillations and the Fermi surface in an underdoped high- $T_{c}$ superconductor. Nature 447, 565-568 (2007).

[9] Yelland, E. A. et al., Quantum Oscillations in the Underdoped Cuprate $\mathrm{YBa}_{2} \mathrm{Cu}_{4} \mathrm{O}_{8}$. arXiv:0707.0057v1 (unpublished).

[10] Abdel-Jawad, M. et al., Anisotropic scattering and anomalous normal-state transport in a high-temperature superconductor. Nature Physics 2, 821-825 (2006).

[11] LeBoeuf, D. et al., Electron pockets in the Fermi surface of hole-doped high- $T_{c}$ superconductors. Nature 450, 533536 (2007).

[12] Anderson, P. W., The Theory of Superconductivity in the High- $T_{c}$ Cuprates (Princeton Univ. Press, 1997).

[13] Emery, V. J. \& Reiter, G., Mechanism for hightemperature superconductivity. Phys. Rev. B 38, 45474556 (1988).

[14] Zhang, F. C. \& Rice, T. M., Effective Hamiltonian for the superconducting Cu oxides. Phys. Rev. B 37, 3759-3761 (1988).

[15] Hozoi, L., Nishimoto, S. \& de Graaf, C., Renormalization 
of quasiparticle hopping integrals by spin interactions in layered copper oxides. Phys. Rev. B 75, 174505 (2007).

[16] Kotliar, G. et al., Electronic structure calculations with dynamical mean-field theory. Rev. Mod. Phys. 78, 865 (2006).

[17] Solovyev, I. V. \& Imada, M., Phys. Rev. B 71, 045103 (2005).

[18] Fulde, P., Wavefunction methods in electronic-structure theory of solids. Adv. Phys. 51, 909-948 (2002).

[19] Helgaker, T., Jørgensen, P. \& Olsen, J., Molecular Electronic-Structure Theory (Wiley, Chichester, 2000).

[20] Roos, B. O., Taylor, P. R. \& Siegbahn, P. E. M., A complete active space SCF method (CASSCF) using a density matrix formulated super-CI approach. Chem. Phys. 48, 157-173 (1980).

[21] de Graaf, C., Local excitations and magnetism in late transition metal oxides, Ph. D. Thesis, University of Groningen, 1998 (available at http://theochem. chem.rug.nl/publications/PDF/ft325

[22] Stoyanova, A., Sousa, C., de Graaf, C. \& Broer, R., Hopping matrix elements from first-principles studies of overlapping fragments: double exchange parameters in manganites. Int. J. Quantum Chem. 106, 2444-2457 (2006).

[23] Capello, M. et al., Variational description of Mott insulators. Phys. Rev. Lett. 94, 026406 (2005).

[24] Pavarini, E. et al., Band-structure trend in hole-doped cuprates and correlation with $T_{c} \max$. Phys. Rev. Lett. 87, 047003 (2001).

[25] Birkenheuer, U., Fulde, P. \& Stoll, H., A simplified method for the computation of correlation effects on the band structure of semiconductors. Theor. Chem. Acc. 116, 398-403 (2006).

[26] Hozoi, L. et al., Ab initio wavefunction-based methods for excited states in solids: correlation corrections to the band structure of ionic oxides. Phys. Rev. B 76, 085109 (2007).

[27] Malmqvist, P.-Å., Calculation of transition density matrices by nonunitary orbital transformations. Int. J. Quantum Chem. 30, 479 (1986).

[28] Andersson, K. et al., Second-order perturbation theory with a CASSCF reference function. Phys. Chem. 94, 5483-5488 (1990).

[29] Smith, M. G. et al., Electron-doped superconductivity at $40 \mathrm{~K}$ in the infinite-layer compound $\mathrm{Sr}_{1-y} \mathrm{Nd}_{y} \mathrm{CuO}_{2}$. Nature 351, 549-551 (1991).

[30] Hozoi, L. \& Laad, M. S., Quasiparticle bands in cuprates by quantum-chemical methods: towards an ab initio description of strong electron correlations. Phys. Rev. Lett. 99256404 (2007).

[31] Ino, A. et al., Electronic structure of $\mathrm{La}_{2-x} \mathrm{Sr}_{x} \mathrm{CuO}_{4}$ in the vicinity of the superconductor-insulator transition. Phys. Rev. B 62, 4137-4141 (2000).

[32] Inosov, D. S. et al., Momentum and energy dependence of the anomalous high-energy dispersion in the electronic structure of high temperature superconductors. Phys. Rev. Lett. 99, 237002 (2007).

[33] Kakehashi, Y. \& Fulde, P., Nonlocal excitation spectra in the two-dimensional doped Hubbard model. J. Phys. Soc. Jpn. 76, 074702 (2007).

[34] Ronning, F. et al., Evolution of a metal to insulator transition in $\mathrm{Ca}_{2-x} \mathrm{Na}_{x} \mathrm{CuO}_{2} \mathrm{Cl}_{2}$ as seen by angle-resolved photoemission. Phys. Rev. B 67, 165101 (2003).

[35] Hashimoto, M. et al., Doping evolution of the electronic structure in the single-layer cuprates $\mathrm{Bi}_{2} \mathrm{Sr}_{2-x} \mathrm{La}_{x} \mathrm{CuO}_{6+\delta}$ : comparison with other single-layer cuprates. arXiv:0801.0782 (unpublished).

[36] Armitage, N. P. et al., Doping dependence of an $n$-type cuprate superconductor investigated by angle-resolved photoemission spectroscopy. Phys. Rev. Lett. 88, 257001 (2002).

[37] Macridin, A. et al., Pseudogap and antiferromagnetic correlations in the Hubbard model. Phys. Rev. Lett. 97, 036401 (2006).

[38] Dopf, G. et al., Direct comparison of angle-resolved photoemission and numerical simulations for high- $T_{c}$ super-

pdf ). conductors. Phys. Rev. Lett. 68, 2082-2085 (1992).

[39] Unger, P. \& Fulde, P., Spectral function of holes in the Emery model. Phys. Rev. B 48, 16607-16622 (1993).

[40] Stojković, B. P. \& Pines, D., Theory of the longitudinal and Hall conductivities of the cuprate superconductors. Phys. Rev. B 55, 8576-8595 (1997).

[41] Yamase, H. \& Metzner, W., Competition of Fermi surface symmetry breaking and superconductivity. Phys. Rev. B 75, 155117 (2007).

[42] Chakravarty, S. \& Kee, H.-Y., Fermi pockets and quantum oscillations of the Hall coefficient in high temperature superconductors. arXiv:0710.0608 (unpublished).

[43] Park, K. \& Sachdev, S., Bond-operator theory of doped antiferromagnets: from Mott insulators with bondcentered charge order to superconductors with nodal fermions. Phys. Rev. B 64, 184510 (2001).

[44] Sutherland, M. et al., Delocalized fermions in underdoped cuprate superconductors. Phys. Rev. Lett. 94, 147004 (2005).

[45] Le Tacon, M. et al., Two energy scales and two distinct quasiparticle dynamics in the superconducting state of underdoped cuprates. Nature Physics 2, 537-543 (2006).

[46] molcas 6, Department of Theoretical Chemistry, University of Lund, Sweden.

[47] Seijo, L., Barandiáran, Z. \& Huzinaga, S., The ab initio model potential method. First series transition metal elements. J. Chem. Phys. 91, 7011-7017 (1989).

[48] Bergner, A. et al., Ab initio energy-adjusted pseudopotentials for elements of groups 13-17. Mol. Phys. 80, 1431-1441 (1993).

[49] Illas, F., Rubio, J. \& Barthelat, J. C., Cu as a oneelectron atom: molecular structure and dissociation energy of CuOH. Chem. Phys. Lett. 119, 397-402 (1985).

[50] Cava, R. J., Santoro, A., Johnson, D. W. \& Rhodes, W. W., Crystal structure of the high-temperature superconductor $\mathrm{La}_{1.85} \mathrm{Sr}_{0.15} \mathrm{CuO}_{4}$ above and below $T_{c}$. Phys. Rev. $B$ 35, 6716-6720 (1987). 